\title{
High energetic and highly stable pulses from a Ho:YLF regenerative amplifier
}

\author{
Peter Kroetz ${ }^{*, a, b}$, Axel Ruehl ${ }^{\mathrm{c}}$, Gourab Chatterjee, ${ }^{\mathrm{a}, \mathrm{b}}$, Anne-Laure Calendron ${ }^{\mathrm{a}, \mathrm{d}, \mathrm{e}}$, Krishna Muraria, ${ }^{\mathrm{c}, \mathrm{d}}$, \\ Huseyin Cankaya ${ }^{\mathrm{a}, \mathrm{c}, \mathrm{e}}$, Franz X. Kärtnera ${ }^{\mathrm{c}, \mathrm{d}, \mathrm{e}}$, Ingmar Hartl' ${ }^{\mathrm{c}}$, and R. J. Dwayne Miller ${ }^{\mathrm{a}, \mathrm{b}, \mathrm{e}, \mathrm{f}}$ \\ ${ }^{a}$ Center for Free-Electron Laser Science (CFEL), Notkestraße 85, D-22607 Hamburg, Germany; \\ ${ }^{b}$ Max-Planck Institute for the Structure and Dynamics of Matter (MPSD), Luruper Chaussee 149, \\ 22761 Hamburg, Germany; ${ }^{c}$ Deutsches Elektronen-Synchrotron (DESY), Notkestrasse 85, 22607 \\ Hamburg, Germany; ${ }^{\mathrm{d} D e p a r t m e n t}$ of Physics, University of Hamburg, 22761 Hamburg, Germany; \\ ${ }^{e}$ Centre for Ultrafast Imaging (CUI), Universität Hamburg, Luruper Chaussee 149, 22761 Hamburg, \\ Germany; ${ }^{f}$ Departments of Chemistry and Physics, University of Toronto, Toronto M5S 1A7, \\ Canada
}

\begin{abstract}
We present results from our Ho:YLF regenerative amplifier (RA) producing up to $6.9 \mathrm{~mJ}$ at a repetition rate of $1 \mathrm{kHz}$ and up to $12.9 \mathrm{~mJ}$ at a repetition rate of $10 \mathrm{~Hz}$. At $1 \mathrm{kHz}$, we observe strongly bifurcating pulses, starting with a certain round trip number, but the measurements identify a highly stable operation point that lies "hidden" beyond the instability region. This operation point allows the extraction of highly stable and high energetic output pulses. Suppression of bifurcation in our system is presented for repetition rates below $750 \mathrm{~Hz}$ and Ho:YLF crystal holder temperatures of 2.5 ${ }^{\circ} \mathrm{C}$.

We furthermore present a stability optimization routine for our Ho:YLF RA that was operated close to gain depletion at a repetition rate of $100 \mathrm{~Hz}$. By varying the Ho:YLF crystal holder temperature the gain depletion level could be fineadjusted, resulting in a highly stable RA system with measured pulse fluctuations of only $0.35 \%$.
\end{abstract}

Keywords: solid-state lasers

\section{INTRODUCTION}

Regenerative amplifiers (RA) were developed shortly after the development of the first laser [1]. Measured energy fluctuations of the output pulses were explained with variations in the shot-to-shot gain due to fluctuations in the pump fluences from the used flashlamps [2]. Nevertheless, to explain the occurrence of instable and rather erratic output pulses, especially at high-repetition-rate RAs [3], fluctuations in the pumping fluences cannot explain all cases of instable output pulse. The appearance of periodic output pulse sequences for consecutive output pulses was related to the life time of the excited ions in the gain media [4]. If the repetition rate of the regenerative amplifier is on the order of the inverse life time or higher, the system becomes susceptible to bifurcating or even multifurcating output pulses.

To generally sketch out an example for bifurcating output pulses from a RA (strictly periodical bistable output pulse energies for certain round trips), Fig. 1 (a) shows simulated output pulse energies as a function of the round trip number at which the pulses are coupled out. The red dots presents the simulation results of a system without any noise from pump and seed source, with a clean bifurcation trajectory for a certain range of round trips (RT) in the middle. For these round trips, the pulses show bistable values, changing strictly periodical between two values. The green dots present simulation results of the same system that is simulated including noise from the pump and seed source. As a consequence, the output pulses at a certain RT are distributed over a certain energy range. In the following, we use the standard deviation of the output pulses as a measure of the output noise at the corresponding RT.

In the following, we refer to the round trips before bifurcation appears, on the left side of the graph, as the first Operation Point (1st OP) and to the round trip where bifurcation again disappears, on the right side of the graph, as the second operation Point (2nd OP). The underlying source for bistable output pulses is a disturbed equilibrium in the gain for

Solid State Lasers XXV: Technology and Devices, edited by W. Andrew Clarkson, Ramesh K. Shori, Proc. of SPIE Vol. 9726, 97260S - (c) 2016 SPIE · CCC code: 0277-786X/16/\$18 · doi: 10.1117/12.2212236 
consecutive pump and amplification cycles, which is sketched out in Fig. 1 (b) for the particular round trip that is marked in (a) with the two stars. As the gain value is periodically changing between two values, the strength of the following amplification process follows, leading to periodically changing pulse energies at the end of the amplification. Although we only present an example for bistable pulses, the type of in-stability is not only restricted to bistability, but can also be multifarious or even completely chaotic [5].

Another important parameter that affects the extend of multifarious output pulses was demonstrated to be the seeding energy of the regenerative amplifier, which can completely suppress this effect when seeding with high pulse energies [5].

Decoupling consecutive pump and amplification cycles, and therefore avoiding the onset of pulse instability, can be achieved by either not depleting the gain media during the pulse amplification process and operating at the 1st OP, or drive the system close to gain saturation during the pumping process and setting the round trip number to sufficiently high values to operate at the 2 nd OP. It has to be noted that not in any case a 2 nd OP exists that allows extraction of high energetic output pulses. This emphasizes the need of simulation tools that help to predict the system behavior of RAs precisely.
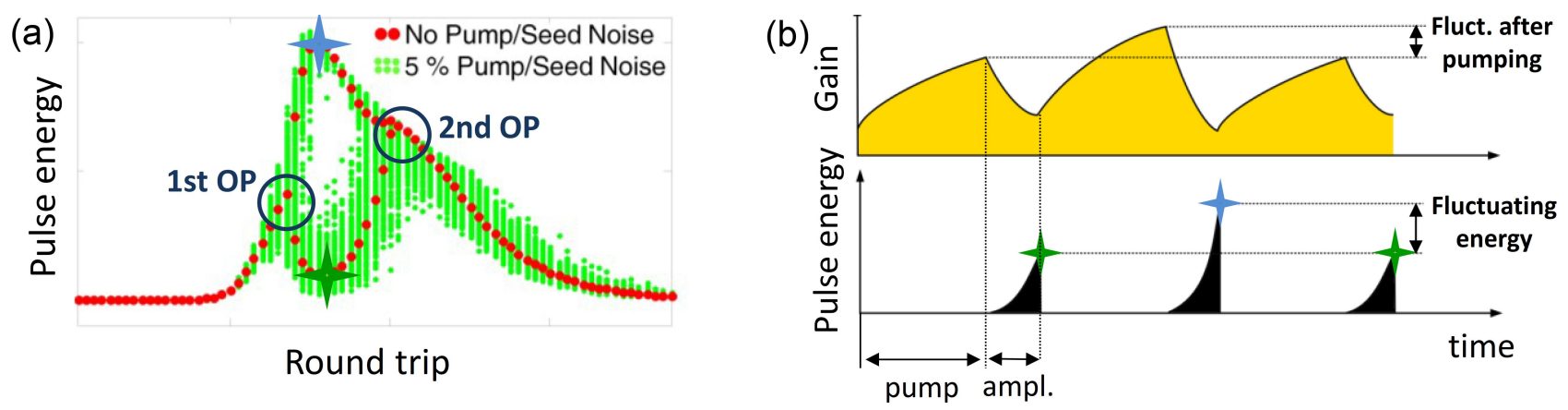

Figure 1. (a) Output pulse energies of a Ho:YLF RA as a function of the round trip, red dots representing the pulse energies if neglecting noise from the pump and seed source. Green dots are simulated taking $5 \%$ noise in pump and seed source into account. Neglecting any noise (red dots), purely single energetic energies can be extracted for round trips up to the 1 st OP and for round trip numbers higher than the 2 nd OP. In between these operation points, the output pulses are periodically changing between two energy values. The underlying source for the bistable output pulses is a disturbed equilibrium in the gain for consecutive pump and amplification cycles, sketched out in (b) for three consecutive cycles. The stars indicate the round trip at which the pulses are coupled out. (b) modified from [4].

In this paper we present measurements for a complete bifurcation trajectory of our Ho:YLF RA, achieving pulse energies of up to $6.9 \mathrm{~mJ}$ at a repetition rate of $1 \mathrm{kHz}$. The measurements, which are in good agreement with simulations, that allow the identification of the 1st OP and 2nd OP, which are separated by bistable output pulses. The prominent advantage of operation at the 2nd OP compared to operation at the 1st OP is a more-than-threefold increase of the pulse energy and a more-than-fivefold decrease in the noise of the output pulses. By lowering the repetition rate, higher pulse energies could be extracted, in particular up to $12.9 \mathrm{~mJ}$ at a repetition rate of $10 \mathrm{~Hz}$. Measurements at lowered repetition rates showed a complete suppression of the onset of bifurcation up to repetition rates of $750 \mathrm{~Hz}$, which exceeds the inverse life-time of Ho:YLF by more than an order of magnitude. This suppression can be explained with stronger gain saturation in the gain medium due to higher pump fluences at lower repetition rates. With a slightly modified RA design we optimized the system stability by tuning the temperature of the Ho:YLF crystal holder, and measured a noise of only $0.35 \%$ (std. dev.) at the highest stability.

To support our measurements, we developed a numerical simulation method, that we modified from classical FrantzNodvik (FN) equations. We used these equations to simulate consecutive pump and amplification cycles, which allows us to include pump and seed noise to the simulations and analyzing the stability and bifurcation behavior of RAs.

\subsection{Simulation approach}

The Frantz-Nodvik (FN) formalism is a common approach to simulate pulse amplification and bases on equations derived by M.L. Frantz and J.S. Nodvik [6]. It is justified for amplification processes in laser gain media as long as the considered time period for the amplification process is short compared to the population life time of the excited ions. The classical Frantz-Nodvik equations are comprehensively explained in Ref [7]. However, here we use a modified version. 
We rephrase the equation that updates the gain to an equation that updates the inversion. The derivation of this equation and its generalization to the spectral regime is shown Ref. [8].

Furthermore, we used a correction term to account for the decay losses of the inversion in the gain medium and the following set of equations can also be used to simulate long cw-pumping processes. For simulating amplification, this correction factor typically can be neglected due to the short amplification time in comparison to the inversion decay time. The only differences in the computation of the pump and seed process are the different values for the absorption and emission cross sections at the considered pump and seed wavelength. To account for the inversion decay losses, we sliced in pump and seed fluence into fluence-slices and consecutively pass the slices through the gain material. Between each passage, the inversion in the gain medium is updated and provides the new gain that the following fluence slice experiences. The slicing of the fluence is also necessary to simulate a homogeneous broadening in Ho:YLF and avoid inhomogeneous broadening effects such as spectral hole burning.

In table 1, we list the main equations needed for our modified FN approach. For comparison, the left column of the table shows the classical FN equations. As the main difference between the two approaches, instead of updating the gain in the classical equations, we modified the equation to update the inversion (Eqn. 6). The classical and the modified FN equations are completely equivalent. The cell in the table marked with * indicates that Eqn. 8, which accounts for the inversion decay losses, is not considered as part of the classical FN equations; however, a comparable expression can also be derived in this case [9]. The main advantage of the modified equation is that it can directly be transferred to the spectral regime without the need of calibrating spectral values with monochromatic values, as necessary with the approach in Ref. [10]. Further information about the simulation approach can be found in Ref. [8].

Table 1: Modified FN equations in direct comparison with our modified equations. The following symbols are used: G, single pass gain; g, gain; $\sigma$, gain cross section; $\sigma_{\text {em }}$, emission cross section; $\sigma_{\text {abs }}$, absorption cross section; $\tau_{\text {gain }}$, excited state life time; $\Delta \mathrm{t}$, time duration of fluence slice; $\mathrm{J}$, fluence; $\mathrm{J}_{\text {stor }}$, stored fluence in gain medium; $\mathrm{N}$, dopant ion density in gain medium; l, length of gain medium; $\mathrm{T}$, single pass transmission; $\mathrm{h}$, planck constant; $\mathrm{c}$, speed of light.

$$
\begin{aligned}
& \text { Classical FN equations } \\
& \text { Modified FN equations } \\
& G_{i-1}=\exp \left(g_{i-1} l\right), \\
& g_{i-1}=\sigma_{g, i-1} N, \\
& \sigma_{g, i-1}=\beta_{i-1}\left(\sigma_{e m}+\sigma_{a b s}\right)-\sigma_{a b s} . \\
& \left.J_{i}=J_{\text {sat }} T \ln \left(1+G_{i-1} \exp \left(\frac{J_{i-1}}{J_{\text {sat }}}\right)-1\right)\right), \\
& J_{s a t}=\frac{h c}{\lambda\left(\sigma_{a b s}+\sigma_{e m}\right)} . \\
& g_{i}=\frac{J_{\text {stor }, i-1}-\left(\frac{J_{i}}{T}-J_{i-1}\right)}{J_{\text {sal }} l}=g_{i-1}-\frac{\frac{J_{i}}{T}-J_{i-1}}{J_{\text {sat }} l}, \\
& J_{\text {stor }, i-1}=J_{\text {sat }} \ln \left(G_{i-1}\right)=\sigma_{g, i-1} N l J_{\text {sat }} . \\
& G_{i-1}=\exp \left(\sigma_{g, i-1} N l\right), \\
& \sigma_{g, i-1}=\beta_{i-1}\left(\sigma_{e m}+\sigma_{a b s}\right)-\sigma_{a b s} . \\
& \left.J_{i}=J_{\text {sat }} T \ln \left(1+G_{i-1} \exp \left(\frac{J_{i-1}}{J_{\text {sat }}}\right)-1\right)\right) . \\
& J_{s a t}=\frac{h c}{\lambda\left(\sigma_{a b s}+\sigma_{e m}\right)} . \\
& \beta_{i}=\beta_{i-1}-\frac{\lambda\left(\frac{J_{i}}{T}-J_{i-1}\right)}{h c l N}, \\
& \beta_{i}^{*}=\beta_{i} \exp \left(-\frac{\Delta t}{\tau_{\text {gain }}}\right)
\end{aligned}
$$




\subsection{Noise study}

To sketch out the capability of the simulation approach, we qualitatively present results for the pulse stability of a Ho:YLF regenerative amplifier as a function of the round trip at which it is coupled out. At the beginning of a simulation, an initial inversion $\beta$ is assumed. To uncouple the simulation results from this initial starting condition, we simulate consecutive pump and amplification cycles. This is sketched out in Fig. 2 (a). This scheme enables us to vary the pump and seed fluence from cycle to cycle and the effect of noise in the pump and seed source on the noise in the output pulses can be analyzed. We define the noise in the pump and seed source as a variation in the total incoming fluence, the value of which is varied at the beginning of each pump and amplification cycle. To analyze the output noise of the RA, we calculate the standard deviation of the output pulse energies for consecutive cycles. Fig. 2 (b) represents a typical noise plot of such a simulation as a function of the round trip at which the pulses are coupled out. The plot reveals a regime that is completely dominated from the pump noise and a regime in which both, pump and seed noise, effect the noise of the output pulses. It is important to note that there is a distinct round trip number where the output noise has a minimum value, which is related to starting gain depletion during amplification (2nd OP).

(a)

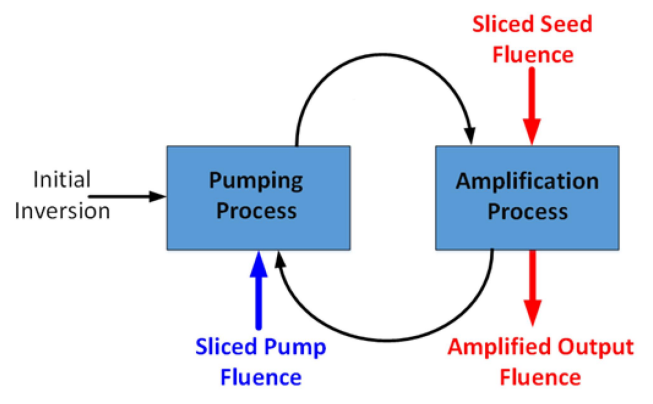

(b)

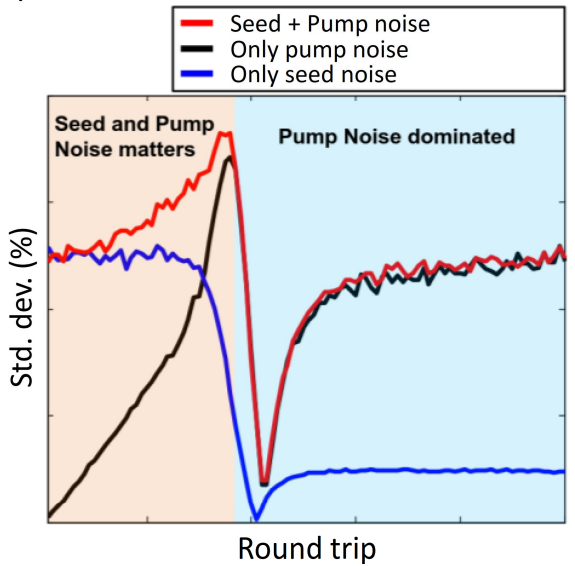

Figure 2: (a) Simulation of consecutive pump and amplification cycles. (b) Qualitative results the output pulse noise of a Ho:YLF regenerative amplifier. The graph shows the different effects of pump and seed noise on the noise of the amplified pulses. 


\section{HO:YLF RA SYSTEM SETUP}

\subsection{Breadboard setup}

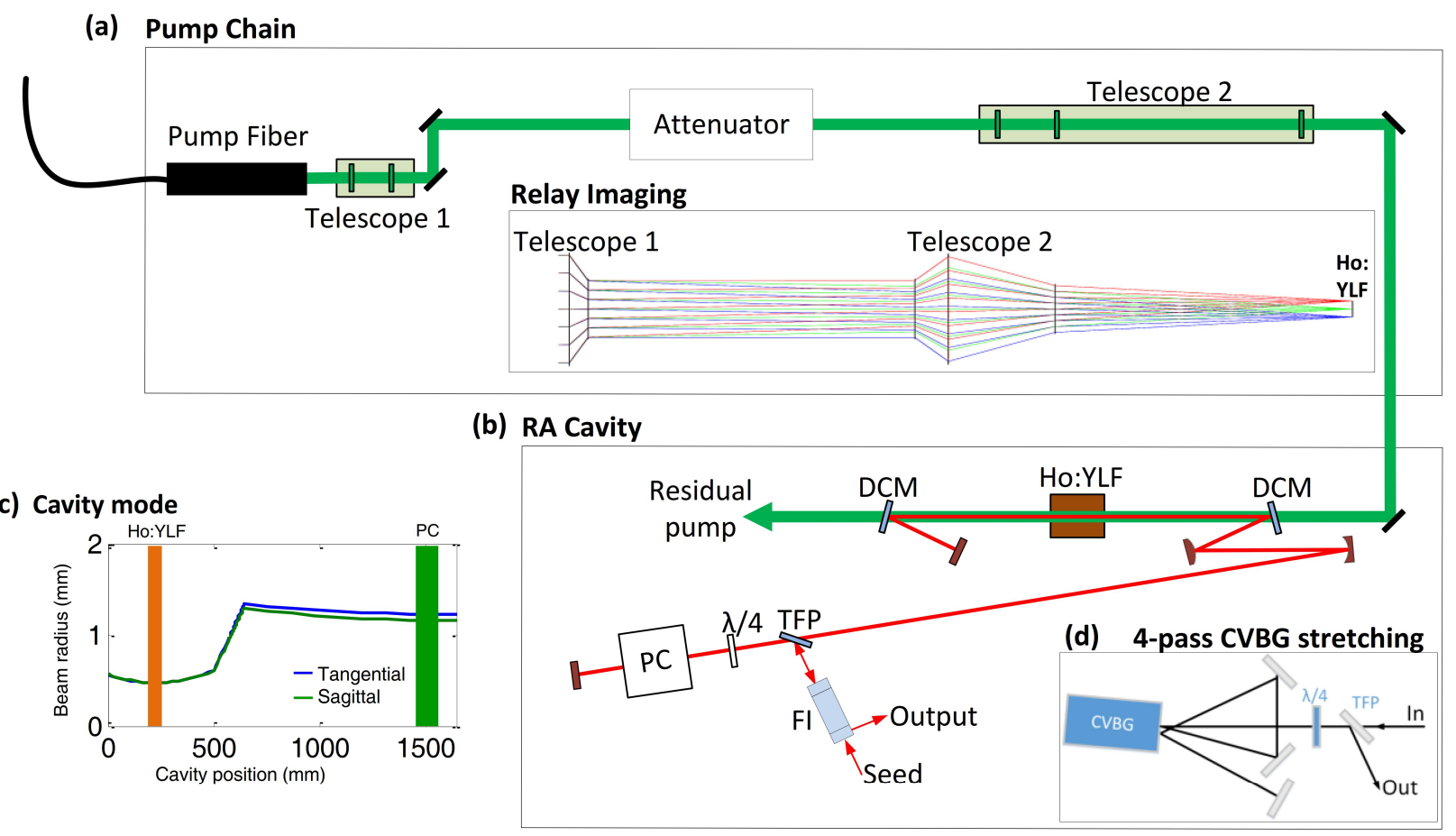

Figure 3: Setup of our laser setup, consisting of (a) a pump chain that images the pump fiber into the Ho:YLF crystal of (b) the Ho:YLF RA cavity. (c) presents the laser cavity mode, which shows only small astigmatism.

Fig. 3 shows the breadboard setup of (a) the pump chain (b) the Ho:YLF RA cavity. The RA cavity is linearly arranged and the mode diameter in the Ho:YLF crystal is $\sim 950 \mu \mathrm{m}$. To minimize the effect of the RTP Pockels cell on the Bintegral, we chose a rather large mode diameter of $2.5 \mathrm{~mm}$ in that part of the cavity. The simulated laser mode is presented in Fig. 3 (c), which exhibits good overlap between the tangential and sagittal mode.

The seed laser was a home-built Ho:fiber soliton mode-locked oscillator that provided $\sim 0.3 \mathrm{~nJ}$ pulses with a spectral bandwidth of $7 \mathrm{~nm}$ [11]. For the stretching of the seed, we used a Chirped Volume Bragg Grating (CVBG) from OptiGrate, with a $10 \mathrm{~mm} \times 10 \mathrm{~mm}$ clear aperture, a chirp factor of $41 \mathrm{ps} / \mathrm{nm}$ and a FWHM bandwidth of $11 \mathrm{~nm}$ around the design wavelength of $2051 \mathrm{~nm}$. Although there is a clear degradation of the spatial beam profile for more than 2 passes through the CVBG, we employ a 4-pass geometry (sketched out in Fig. 4 (d)) to achieve highly chirped pulses, leading with the CVBG's specified diffraction efficiency of $90 \%$ to a theoretical streching loss of at least $34.4 \%$. For the in- and out-coupling of the pulses, we employed a 6-mm aperture RTP pockels cell (Leysop), a fast Pockels cell driver with rise- and fall-times of $<4.5 \mathrm{~ns}$ (BME Bergmann), and an 8-mm aperture Faraday rotator (FastPulse Technology, Inc.).

The pump chain consists of a polarization splitting attenuator and a zoom-telescope relay imaging system. The telescope system, first, images the end of the pump fiber into the Ho:YLF crystal and, second, matches the pump spot size to the cavity mode spot size. The polarization splitting attenuator polarizes the randomly polarized pump into two arms which can then be separately attenuated (not shown in the figure). The two individually attenuable polarizations are then aligned and overlaid again to achieve a single pump spot in the gain crystal. This polarizing splitting attenuator enables a safe alignment of the system with low pump powers. We use a rather short Ho:YLF crystal with a length of $9 \mathrm{~mm}$ and with $1 \%$ doping, which is reflected in a modest $\mathrm{CW}$-output power of up to $14.5 \mathrm{~W}$ despite the $108 \mathrm{~W}$ of incident pump power. A flexible cooling of the Ho:YLF crystal was achieved with an electrically cooled Peltier element. 


\subsection{Experimental identification of 2nd OP}

Fig. 4 (b) presents output pulse energies measured with our Ho:YLF RA as a function of the round trip number. The system was operated at a repetition rate of $1 \mathrm{kHz}$, and the crystal holder temperature was kept at $2.5^{\circ} \mathrm{C}$. For low round trip numbers, we measured quasi single energetic output pulses up to round trip 22. For round trip 23, we observe the onset of bifurcation, resulting in bistable output pulses up to round trip 33. For the round trips $>33$, bifurcation disappeared and the RA again produces single energetic output pulses. We define 1st OP as the round trip before the output pulse noise exceeds $10 \%$ (std. dev.) before the onset of bifurcation. As this definition bases on a threshold value (in this case $10 \%$ ), also other definitions, depending on the sensitivity of certain applications on the noise level could be chosen. Regarding the 2nd OP it is possible to find a more concrete definition for the 2nd OP, which we define in the following as the round trip at which the output pulse noise has its minimum. Comparing 1st OP and 2nd OP, we find not only an almost threefold increase of the pulse energy ( $2.4 \mathrm{~mJ}$ vs. $6.9 \mathrm{~mJ})$ but also a more than fivefold reduction of the output pulse noise ( $6 \%$ vs $1.1 \%$ ). The simulations for this system, presented in Fig. 4 (a), show good agreement with the measurements. The spectral width of the output pulses at the 2nd OP was measured to be $2.3 \mathrm{~nm}$ (see Fig. 4 (c)) corresponding to a calculated Fourier limited pulse duration of $1.9 \mathrm{ps}$. These results were already presented in Ref. [12].
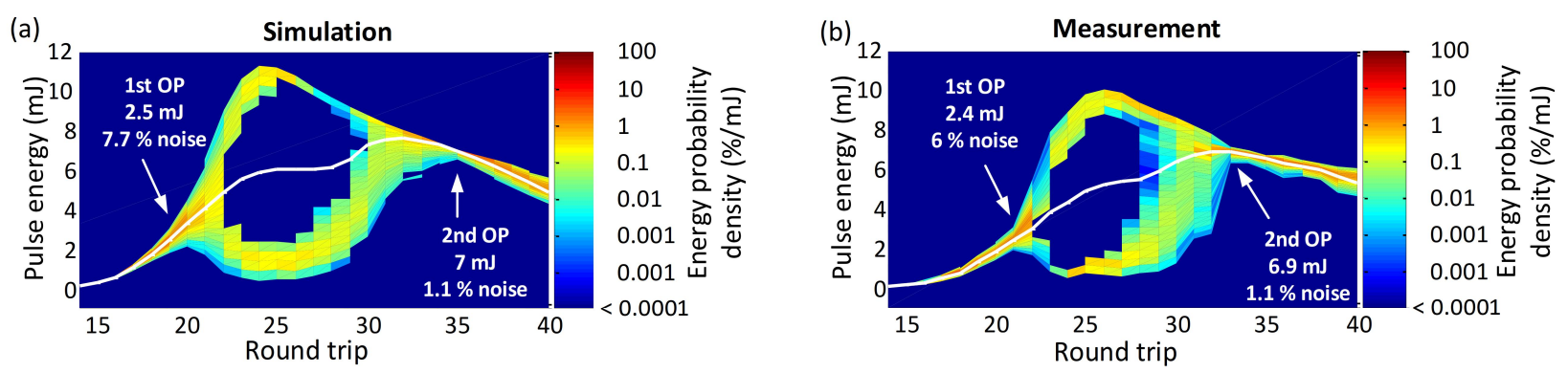

Figure 4: (a) simulated and (b) measured bifurcation trajectory as a function of the round trip at which the laser pulses are coupled out of the RA cavity. Operation at the 2nd OP shows significantly higher pulse energies and lower noise values than operation at the 1 st OP. Data from Ref. [12].

\subsection{System performance in dependence of repetition rate}

To further characterize the performance the Ho:YLF RA, we varied the repetition rate between $1 \mathrm{kHz}$ and $10 \mathrm{~Hz}$, while keeping the incident pump power on the crystal at $108 \mathrm{~W}$. Decreasing the repetition rate results in higher pump fluences per pumping cycle and consequently in a higher gain and higher output pulse energies, which is presented in Fig. 5 as a function of the round trip number. Although the pump fluence increases by a factor of 100 (comparing the pump fluence at $1 \mathrm{kHz}$ with the pump fluence at $10 \mathrm{~Hz}$ ), the maximum extractable pulse energy only increases by a factor less than two $(6.9 \mathrm{~mJ}$ at $1 \mathrm{kHz}$ vs. $12.9 \mathrm{~mJ}$ at $10 \mathrm{~Hz})$. This is a strong indication for gain saturation achieved at lower repetition rates. The max. peak fluence in the gain crystal was up to $3.7 \mathrm{~J} / \mathrm{cm}^{2}$, at $10 \mathrm{~Hz}$ and a beam diameter in the Ho:YLF crystal of $950 \mu \mathrm{m}$.

Due to the high stretching of the seed pulses, the B-Integral of the amplified pulses is estimated to remain between 1 and 1.4 for pulses that we assumed to be stretched to a duration of $377 \mathrm{ps}$. This is based on the specifications of the CVBG (4 CVBG passes, $2.3 \mathrm{~nm}$ bandwidth, $41 \mathrm{ps} / \mathrm{nm}$ stretching ratio). For the calculation of the B-Integral, it is important to note, that due to the unknown value for the non-linear refractive index of the RTP Pockels cell, the value of its isomorph KTP $\left(12^{*} 10^{\wedge}(-16)\right)$ is used [13], which has similar linear and non-linear optical properties [14]. Under this assumption, approximately $75 \%$ of the total B-Integral is contributed from the Pockels cell.

Comparing the beam shape at lower repetition rates, we observe strong beam distortions, for higher repetition rates. This is also shown in Fig. 5 for the repetition rates $1 \mathrm{kHz}, 250 \mathrm{~Hz}$ and $10 \mathrm{~Hz}$, recorded at the max. pulse energy. This effect might be related to the high average output power at $1 \mathrm{kHz}$, as we also observe beam distortions in $\mathrm{CW}$ operation for high intra-cavity powers (low output coupling losses). 


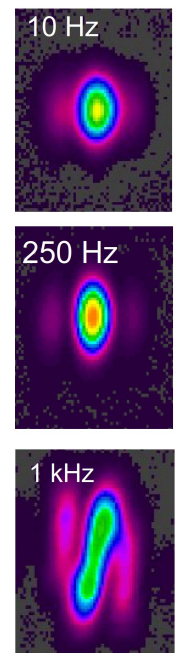

Figure 5: Pulse energy as a function of the round trip number for repetition rates between $1 \mathrm{kHz}$ and $10 \mathrm{~Hz}$. The up-scaling of the pulse energy is limited by gain saturation at lower repetition rates.

\subsection{Suppression of bifurcation}

In our measurements, two parameters showed a significant effect on the bifurcation susceptibility of our RA system. First, reducing the repetition rate below $750 \mathrm{~Hz}$ leads to complete absence of any bifurcation in the output pulses at any round trip. This is presented in Fig. 6 (a). While there is strong bifurcation present for $1 \mathrm{kHz}$ and $850 \mathrm{~Hz}$, indicated by the sudden increase in the pulse noise around round trip 22, no such a noise increase is present in the curve for $750 \mathrm{~Hz}$, and also for the repetition rates below (not shown in the graph). We attribute this behavior to increased gain saturation, due to the increased pump fluence, at lower repetition rates. Second, for a crystal holder temperature of $2.5^{\circ} \mathrm{C}$, we also observe a complete suppression of any bifurcation, while bifurcating pulses appear in the system for the higher temperatures $12{ }^{\circ} \mathrm{C}$ and $19{ }^{\circ} \mathrm{C}$, presented in Fig. 6 (b). Again, this can be explained with higher gain saturation, but this time due to higher absorbed pump powers at lower crystal temperatures. Furthermore, while decreasing the temperature to $2.5^{\circ} \mathrm{C}$, the max. output pulse energy is increasing by $33 \%$, compared to operation at $19{ }^{\circ} \mathrm{C}$. 
(a)

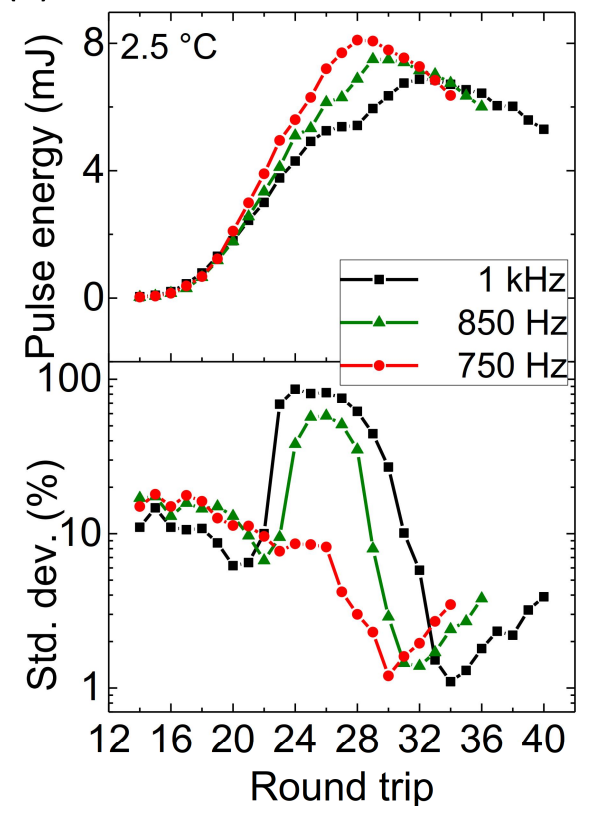

(b)

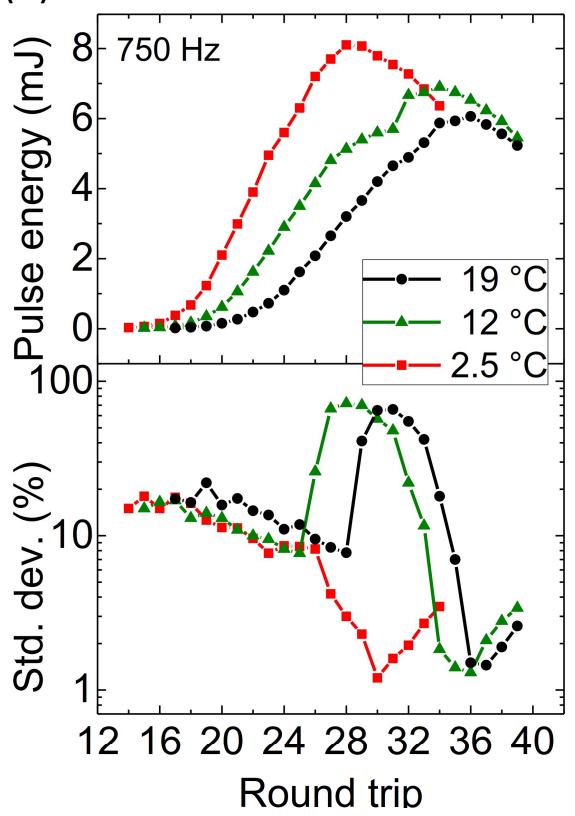

Figure 6. (a) Pulse energy and output noise (std. dev.) as a function of the round trip. While for $750 \mathrm{~Hz}$, there is no sign of bifurcation, it appears for higher repetition rates. (b) Pulse energy and output noise (std. dev.) as a function of the Ho:YLF crystal holder temperature. While there is no sign for bifurcation for the lowest temperature of $2.5^{\circ} \mathrm{C}$, for higher temperatures it appears.

\section{STABILITY OPTIMIZATION AT 2ND OP}

For the following measurement, we slightly changed our Ho:YFL setup. We use a Ho:YLF crystal with a length of 2.1 $\mathrm{cm}$ and $1 \%$ doping and changed the stretching and compression setup to a double-pass configuration employing the same CVBG. We measured compression losses of $30 \%$ with this configuration. To avoid the onset of bifurcation, we limit the repetition rate to $100 \mathrm{~Hz}$ and pump the system with moderate pumping powers of $\sim 22 \mathrm{~W}$. To operate the RA at the 2nd OP with highest stability, our simulations predict a specific gain depletion value after the pulse amplification that leads to the highest stable output pulses. To operate the RA at this highest stable point, we chose to vary the temperature of the Ho:YLF crystal holder. The temperature has a strong effect on the gain a pulse experiences during amplification. Therefore, varying the temperature will result in varying gain depletion levels if the pulses are coupled out at a fixed round trip. Fig. 7 presents the measured standard deviation of the output pulses as a function of the crystal holder temperature.

For the following measurements, the amplified pulses were always switched out after 27 round trips. With a crystal holder temperature of $-10^{\circ} \mathrm{C}$, the RA was operated about 6 RTs after the 2nd OP. As the simulations predict the optimum depletion level closer to the 2 nd OP, the gain could be called "over-depleted". At a temperature of $18{ }^{\circ} \mathrm{C}$, the RA was operated about $1 \mathrm{RT}$ before the 2 nd OP. At each temperature $>3000$ consecutive output pulses were recorded with a commercially calibrated energy meter (Coherent) and used to calculate the standard deviation as a measure for the pulse stability. The lowest noise was measured to be $0.35 \%$ at a temperature of $5{ }^{\circ} \mathrm{C}$, while the noise significantly increased for lower and higher temperatures. The pulse energies shown in Fig. 7 are representing the pulse energies of the already compressed output pulses. 


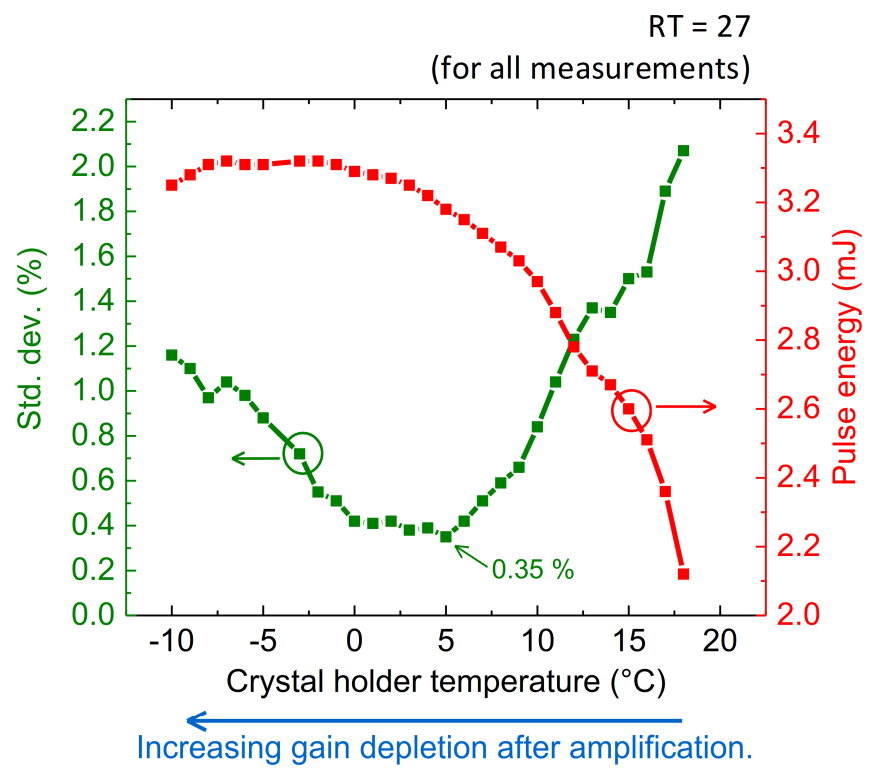

Figure 7. Pulse noise and compressed output pulse energy as a function of the Ho:YLF crystal holder temperature. For a temperature of $5^{\circ} \mathrm{C}$, we measured the highest stability with $0.35 \%$ (std. dev.).

\section{OUTLOOK}

To decrease the sensitivity of the cavity mode to thermal lensing in the Ho:YLF crystal, the cavity can be extended at both ends to a double-folded z-cavity, which shows significantly improved thermal stability compared to the current design [15]. To furthermore improve the Ho:YLF RA, especially at higher pumping powers, we currently reworking our crystal holder and TEC-cooling, to more cool the laser crystal further. We can envisage that these measures will increase the repetition rate without bifurcation to higher values, increase further the maximum extractable pulse energy and improve the spatial beam profile.

\section{CONCLUSIONS}

We presented a complete bifurcation trajectory measured with our Ho:YLF RA at $1 \mathrm{kHz}$, as a function of the round trip number. We identified an operation point (2nd OP) allowing the extraction of highly stable and high energetic laser pulses. The measurements show that this operation point lies "hidden" at higher round trip numbers than the onset of bifurcation. At a repetition rate of $1 \mathrm{kHz}$, we could extract pulse energies of up to $6.9 \mathrm{~mJ}$. For lower repetition rates, the pulse energies increase due to higher pumping fluences, resulting in up to $12.9 \mathrm{~mJ}$ at $10 \mathrm{~Hz}$. The onset of bifurcation could be suppressed up to repetition rates of $750 \mathrm{~Hz}$, which is more than an order of magnitude higher than the inverse life time of Ho:YLF. We observed a high temperature dependence of the system performance. While for the lowest temperature of $2.5^{\circ} \mathrm{C}$, bifurcation was completely suppressed, it appeared for higher temperatures again. In both cases, we attribute the suppression of bifurcation to an increasing gain saturation in the Ho:YLF crystals.

To realize the highest stable system operation, we optimized gain depletion during amplification via varying the temperature of the crystal holder. For a temperature of $5{ }^{\circ} \mathrm{C}$, we measured the highest stability with pulse fluctuations of only $0.35 \%$.

\section{REFERENCES}

[1] J.E. Swain, F. Rainer, “Many-Pass Resonant Laser Amplifier,” IEEE J. Quantum Electronics 5(7), 385-386 (1969).

[2] J.E. Murray, W.H. Lowdermilk, “ND:YAG regenerative amplifier,” J. Appl. Phys. 51(7),3549-3555 (1980). 
[3] X.D.Wang, P. Basséras, J. Sweetser, I.A. Walmsley, and R.J.D. Miller, "Regenerative pulse amplification in the 10-kHz range," Opt. Lett. 15(15), 839-841 (1990).

[4] J. Dörring, A. Killi, A. Lang, M. Lederer, D. Kopf, and U. Morgner, "Periodic doubling and deterministic chaos in continuously pumped regenerative amplifiers," Opt. Expr. 12(8), 1759-1767 (2004).

[5] M. Grishin, V. Gulbinas, A. Michailovas, "Dynamics of high repetition rate regenerative amplifiers," Opt. Expr. 15(15), 9434-9442 (2007).

[6] L. M. Frantz and J. S. Nodvik, "Theory of pulse propagation in a laser amplifier," J. Appl. Phys. 34, 2346-2349 (1963).

[7] W. Koechner, [Solid-State Laser Engineering], Springer-Verlag, (1996).

[8] Peter Kroetz, Max Planck Institute for the Dynamics and Structure of Matter (MPSD), Luruper Chaussee 149, 22761 Hamburg, and Axel Ruehl, Krishna Murari, Huseyin Cankaya, Franz X. Kärtner, Ingmar Hartl, and R. J. Dwayne Miller are preparing a manuscript to be called " Numerical study of spectral shaping in high energy Ho:YLF amplifiers."

[9] Haider Zia, "Experimental and Theoretical Studies in Non-Linear Optical Applications: Fiber Oscillators, Regenerative Amplifiers, Simulations on White-Light Generation," Ph.D. thesis (University Hamburg, 2015).

[10] Pierre Raybaut, François Balembois, Frédéric Druon, and Patrick Georges, "Numerical and Experimental Study of Gain Narrowing in Ytterbium-Based Regenerative Amplifiers," IEEE J. Quantum Electron. 41, 415-425 (2005).

[11]Peng Li, Axel Ruehl, Coleen Bransley, Ingmar Hartl, "Low Noise, tunable Ho:fiber Soliton Oscillator for Ho:YLF Amplifier Seeding,” arXiv:1509.09184 (2015).

[12] Peter Kroetz, Axel Ruehl, Gourab Chatterjee, Anne-Laure Calendron, Krishna Murari, Huseyin Cankaya, Peng Li, Franz X. Kärtner, Ingmar Hartl, and R. J. Dwayne Miller, "Overcoming bifurcation instability in highrepetition-rate Ho:YLF regenerative amplifiers," Opt. Lett. 40, 5427-5430 (2015).

[13]H. P. Li, C. H. Kam, Y. L. Lam, and W. Ji, "Femtosecond Z-scan measurements of nonlinear refraction in nonlinear optical crystals," Opt. Mater. 15, 237 (2000).

[14]L.-T. Cheng, L. K. Cheng, and J. D. Bierlein, "Linear and Nonlinear Optical Properties of the Arsenate Isomorphs of KTP," Proc. SPIE 1863, 43 (1993).

[15] Peter Kroetz, A. Ruehl, K. Murari, H. Cankaya, A. Calendron, F. Kaertner, I. Hartl, and R. J. D. Miller, "High Energy and Low Noise Ho:YLF Regenerative Amplifiers: A Noise and Stability Analysis," in CLEO:2015, OSA Technical Digest (online) (Optical Society of America, 2015), paper SF1F.3. 